\title{
High-Performance Condenser Microphone with Fully Integrated CMOS Amplifier and DC-DC Voltage Converter
}

\author{
Michael Pedersen, Wouter Olthuis, and Piet Bergveld
}

\begin{abstract}
The development of a capacitive microphone with an integrated detection circuit is described. The condenser microphone is made by micromachining of polyimide on silicon. Therefore, the structure can be realized by postprocessing on substrates containing integrated circuits (IC's), independently of the IC process. Integrated microphones with excellent performances have been realized on a CMOS substrate containing dc-dc voltage converters and preamplifiers. The measured sensitivity of the integrated condenser microphone was $10 \mathrm{mV} / \mathrm{Pa}$, and the equivalent noise level (ENL) was $27 \mathrm{~dB}(\mathrm{~A})$ re. $20 \mu \mathrm{Pa}$ for a power supply voltage of $1.9 \mathrm{~V}$, which was measured with no bias voltage applied to the microphone. Furthermore, a back chamber of infinite volume was used in all reported measurements and simulations. [338]
\end{abstract}

Index Terms-Capacitance transducers, integrated electronics, microphones, polyimide films, voltage multipliers.

\section{INTRODUCTION}

$\mathbf{T}$ HE EVOLUTION of a new generation of small-size high-performance microphones based on silicon micromachining has until now been focused on the need to monolithically integrate the sensor structure with an electronic circuit to maximize the performance. The integration is needed to improve the sensitivity and noise level of the microphone to make it competitive with existing devices. Furthermore, the application of silicon condenser microphones with an integrated dc-dc voltage converter for biasing the device could be favorable to conventional electret microphones, whereby the long-term drift problems associated with electrets could be eliminated. Considering, however, the silicon condenser microphone technologies described so far in the literature [1]-[8], they all contain deposition, etching, and/or bonding processes, which are not directly compatible with integrated circuit (IC) technology. Furthermore, only two integrated condenser microphones have been suggested [9], [10], which were both made by merging micromachining and IC processing. To realize a more flexible approach in which the microphone can be produced independently of the electronic circuitry, new

Manuscript received March 11, 1998; revised August 12, 1998. This work was supported by the Dutch Technical Foundation (STW). Subject Editor, G. Stemme.

M. Pedersen was with the MESA Research Institute, University of Twente, NL-7500 AE Enschede, The Netherlands. He is now with Knowles Electronics, Rolling Meadows, IL 60008 USA.

W. Olthuis and P. Bergveld are with the MESA Research Institute, University of Twente, NL-7500 AE Enschede, The Netherlands.

Publisher Item Identifier S 1057-7157(98)08557-6. developments have been required to attain a reliable process that will not affect the integrity of the electronic circuitry. For this purpose, the sensor structure shown in Fig. 1 has been developed [11]. As opposed to more conventional silicon sensors, this sensor is a plastic (polyimide) structure made directly on the substrate by micromachining. The advantage of this structure is the low-temperature fabrication process $\left(<300^{\circ} \mathrm{C}\right)$, which can be performed on substrates containing IC's without inflicting any damage or parameter shifts in the circuits. Furthermore, by changing the troublesome bulk etching of the silicon substrate from $\mathrm{KOH}$ to deep reactive ion etching, much better process reliability has been achieved.

\section{Design of the InTEgrated Condenser Microphone}

The schematic diagram of the integrated condenser microphone is shown in Fig. 2. The device comprises a Dicksontype dc-dc voltage converter [12], condenser microphone, and PMOS buffer amplifier. The voltage converter is basically a charge pump in which charge is built up at the output by the two-phase oscillator. The diodes in this design of the voltage converter have been implemented by using the drain-bulk (pn) junction in a p-type MOSFET, which provides much better efficiency of the converter compared to previous designs with n-type MOSFET's coupled as diodes, the major reason being the elimination of the bulk effect. In the circuit in Fig. 2, it can be seen that the voltage converter does not have to provide any output current since the output is decoupled by the microphone. In this situation, the output voltage of the converter is given by [12]

$$
V_{\mathrm{dc}, \text { out }}=V_{b}+N\left(\frac{C}{C+C_{p}} V_{b}-V_{d}\right)-V_{d}
$$

where $V_{b}$ is the supply voltage, $N$ is the number of stages in the voltage converter, $C$ is the value of the capacitor in each stage, $C_{p}$ is the parasitic capacitance in each stage, and $V_{d}$ is the voltage drop over the diodes. In the implemented ten-stage converter, the values are [13]: $C=10 \mathrm{pF}, C_{p}=0.6$ $\mathrm{pF} / \mathrm{stage}$, and $V_{d}=0.5 \mathrm{~V}$, yielding an output voltage of 14.3 $\mathrm{V}$ for a supply voltage of $1.9 \mathrm{~V}$. The power consumption of the dc-dc converter has been measured to be $110 \mu \mathrm{W}$ at a supply voltage of $2.4 \mathrm{~V}$. The output noise of the converter has not been measured and is expected to be concentrated around the frequency of the oscillator $(2 \mathrm{MHz})$. Considering the A weighting normally used in acoustical noise measurements, 


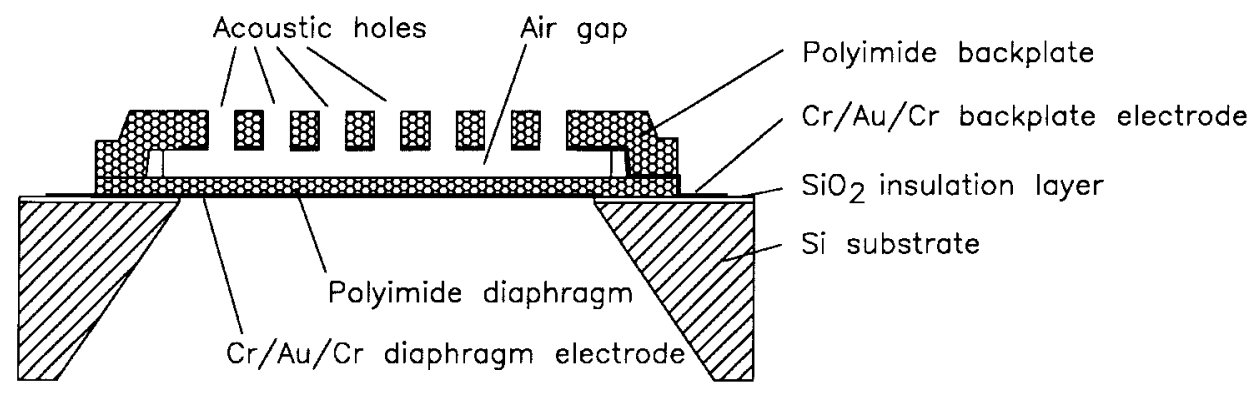

Fig. 1. Cross-sectional view of the polyimide condenser microphone.

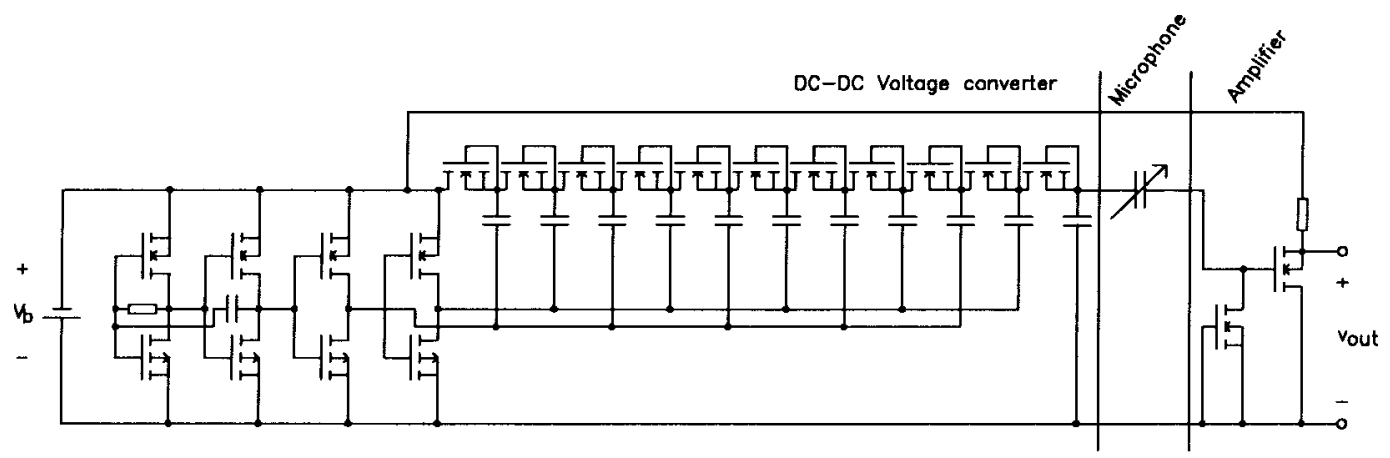

Fig. 2. Schematic diagram of the integrated condenser microphone with dc-dc voltage converter and preamplifier.

this noise is not expected to be of any influence. The only concern is to avoid oversteering the buffer amplifier with the high-frequency signal.

The buffer amplifier is a simple source follower in which the same type of diode as for the voltage converter is used to ensure the dc potential of the input is grounded, whereby the output voltage of the converter will appear over the microphone. P-type MOSFET's were preferred since they have slightly lower noise than n-type devices, and the dimensions and source resistance were optimized to achieve the best balance between noise and power consumption [13]. The implemented preamplifier has a P-type MOSFET with dimensions (W/L) 660/5 $\mu \mathrm{m}$ and a source resistor of $20 \mathrm{k} \Omega$, yielding a gain of $-0.4 \mathrm{~dB}$. For a supply voltage of $2.4 \mathrm{~V}$, the power consumption was measured to be $188 \mu \mathrm{W}$, and the A-weighted input noise was $3.6 \mu \mathrm{V}$.

The behavior of the condenser microphone itself is described by the electromechanical circuit shown in Fig. 3. As illustrated previously [11], the mechanical behavior of the microphone can be described by the mechanical part of the circuit [Fig. 3(a)], which relates forces (potential) and velocities (flow) in the microphone structure. For a detailed description of this circuit, which includes radiation and compliance of the diaphragm, air-streaming resistance in the air gap and acoustic holes in backplate, and compliance of the backplate and backchamber, the reader should refer to [11]. The mechanical components are given by the following relations.

Diaphragm radiation:

$$
R_{r(\omega)}=\frac{0.1886}{\pi^{3}} \frac{L^{6} \rho_{0} \omega^{4}}{c^{3}} \quad M_{r}=\frac{2.67 L^{3} \rho_{0}}{\pi \sqrt{\pi}}
$$

Diaphragm compliance and mass inertia:

$$
C_{d}=\frac{1}{30 \sigma_{d} h_{d}} \quad M_{d}=\rho_{d} h_{d} L^{2}
$$

Backplate compliance and mass inertia:

$$
C_{b}=\frac{1}{30 \sigma_{b} h_{b} \sqrt{1-\frac{4 a^{2}}{b^{2}}}} \quad M_{b}=\rho_{b} h_{b} L^{2} \sqrt{1-\frac{4 a^{2}}{b^{2}}} .
$$

Air-gap streaming resistance and mass inertia:

$$
R_{a}=\frac{1.22 \eta \pi L^{2} b^{2}}{h_{a}^{3}} B \quad M_{a}=\frac{0.102 \rho_{0} \pi L^{2} b^{2}}{h_{a}} B .
$$

Acoustic holes streaming resistance and mass inertia:

$$
R_{h}=\frac{12 \eta h_{b} L^{2}}{b^{2}} \quad M_{h}=\frac{24 \rho_{0} h_{b} a^{2} L^{2}}{5 b^{2}} .
$$

Compliance of microphone backchamber:

$$
C_{b c}=\frac{V}{\rho_{0} c^{2} L^{4}} .
$$

In these equations, $L$ is the side length of the microphone, $\rho_{0}$ is the density of air, $c$ is the speed of sound in air, $\omega$ is the angular frequency, $\sigma_{d}, h_{d}$, and $\rho_{d}$ are the intrinsic stress, thickness, and density of the diaphragm, $\sigma_{b}, h_{b}$, and $\rho_{b}$ are the intrinsic stress, thickness, and density of the backplate, $2 a$ and $b$ are the size and center-to-center distance of the square acoustic holes in the backplate, $\eta$ is the dynamic viscosity of air, $h_{a}$ is the height of the air gap, $V$ is the volume of the backchamber, and $B$ is a value given by

$$
B=\frac{1}{4} \ln \left(\frac{0.160 b^{2}}{a^{2}}\right)-\frac{3}{8}+3.133 \frac{a^{2}}{b^{2}}-4.907 \frac{a^{4}}{b^{4}} .
$$




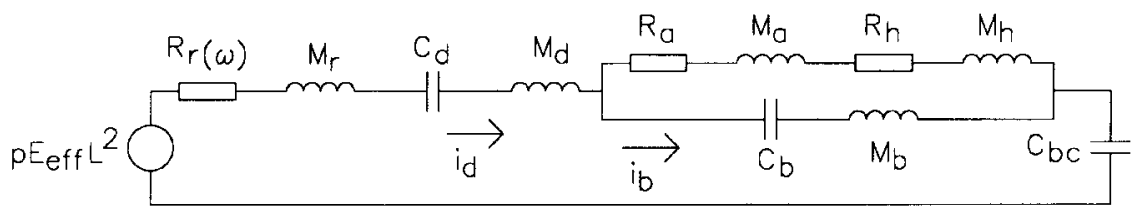

(a)

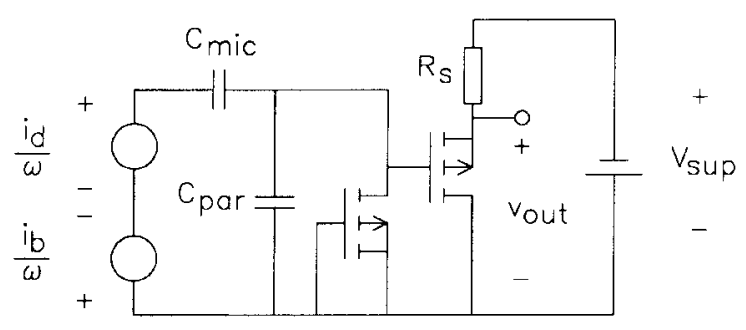

(b)

Fig. 3. Electromechanical diagram of the condenser microphone and preamplifier. $E_{\text {eff }}$ is the effective electrical field in the airgap from (9), $i_{d}$ is the velocity of the diaphragm, and $i_{b}$ is the velocity of the backplate.

For harmonic sound pressures, the displacement of the diaphragm and the backplate can be derived simply by dividing the velocities of the diaphragm $i_{d}$ and the backplate and $i_{b}$ [see Fig. 3(a)] by the angular frequency $\omega$ of the sound pressure. For frequencies below the first resonance of the diaphragm and the backplate, the phase shift between the two plates will be less than $180^{\circ}$, and, thus, the effective change of the air gap between the diaphragm and backplate can be calculated by subtracting the two deflections, as indicated by the polarity of the two voltage sources in Fig. 3(b). The open-circuit electrical response of the microphone is derived by multiplying the change of the air gap by the effective electrical field in the air gap. For the integrated microphone with the structure shown in Fig. 1, the effective electrical field is given by

$$
E_{\text {eff }}=\left(1-\frac{4 a^{2}}{b^{2}}\right) \frac{\varepsilon_{d}}{\varepsilon_{d} h_{a}+h_{d}} V_{\text {dc,out }} \quad\left(h_{a} \ll 2 a\right)
$$

where $\varepsilon_{d}$ is the relative permittivity of the diaphragm material and $V_{\mathrm{dc} \text {,out }}$ is the output voltage of the voltage converter connected to the microphone. In the simulation, the multiplication of the effective electrical field is done directly by scaling the mechanical source in Fig. 3(a), whereby the two voltage sources in Fig. 3(b) represent the true open-circuit electrical output of the microphone.

The microphone is connected to a preamplifier, as shown in the electrical circuit in Fig. 3(b). Assuming charge conservation in the microphone, the dc-dc voltage converter and variable microphone capacitance in Fig. 2 can be replaced by the two voltage sources $i_{d} / \omega$ and $i_{b} / \omega$ and a capacitive output impedance $C_{\text {mic }}$ given by

$$
C_{\text {mic }}=\left(1-\frac{4 a^{2}}{b^{2}}\right) \frac{\varepsilon_{d} \varepsilon_{0} L^{2}}{\varepsilon_{d} h_{a}+h_{d}} \quad\left(h_{a} \ll 2 a\right)
$$

in which $\varepsilon_{0}$ is the absolute permittivity in vacuum. The microphone is loaded electrically by a parasitic capacitance $C_{\text {par }}$ due to the interconnections and internal parasitics in the microphone structure. With this circuit, the response of the integrated microphone can be simulated, and by introducing the relevant noise sources in the circuit, the input noise of the microphone can also be simulated [13]. The overall input
TABLE I

Material Parameters and Microphone Dimensions

\begin{tabular}{ll}
\hline Intrinsic tensile stress $\sigma$ (diaphragm/backplate) & $20 \mathrm{MPa}$ \\
Density $\rho$ (diaphragm/backplate) & $1430 \mathrm{~kg} / \mathrm{m}^{3}$ \\
Relative permittivity $\varepsilon_{d}$ (diaphragm) & 3.4 \\
Thickness of diaphragm $h_{d}$ & $1.1 \mu \mathrm{m}$ \\
Thickness of backplate $h_{b}$ & $15 \mu \mathrm{m}$ \\
Air gap $h_{a}$ & $3.6 \mu \mathrm{m}$ \\
Side length $L$ & $2.2 \mathrm{~mm}$ \\
Acoustic hole size in backplate $2 a$ & $30 \times 30 \mu \mathrm{m}$ \\
Acoustic hole center-to-center distance $b$ & $80 \mu \mathrm{m}$ \\
\hline
\end{tabular}

noise is a combination of the noise in the preamplifier and the thermal noise in the microphone itself.

Also, in a condenser microphone, the question of stability is important since the electrostatic attraction forces between the diaphragm and the backplate, induced by the dc bias voltage, may cause the structure to collapse. The nature of this problem must be known to find a limitation of the dc bias voltage, preventing the situation of collapse. A detailed description of this complicated nonlinear problem can be found elsewhere [13], [14] in which it was shown that the quasi-static deflection of the diaphragm and backplate can be described by a nonlinear-coupled differential equation system, the stability of which coincides with the stability of the microphone. From this quasi-static model, the critical bias voltage (CBV) can be found above which the microphone structure will be collapsed.

The dimensions of the integrated polyimide microphone were optimized for performance (sensitivity and noise level) and critical bias voltage, yielding the values given in Table I. Also listed are the relevant material parameters used for the calculations with the dynamic model described above and the quasi-static stability model [14]. The integrated microphone with these dimensions was calculated to have a critical bias voltage of $24 \mathrm{~V}$, a 3-dB bandwidth of $27 \mathrm{kHz}$, and a sensitivity of $29.1 \mathrm{mV} / \mathrm{Pa}$ for a supply voltage of $1.9 \mathrm{~V}$.

\section{FABRICATION PROCESS}

The fabrication process of the integrated microphone is shown in Fig. 4. First, the electrical circuits are formed using 


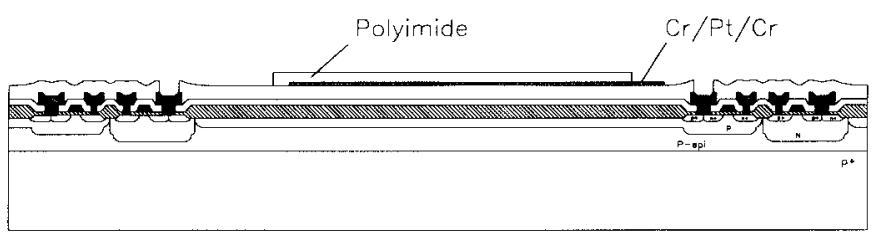

(a)

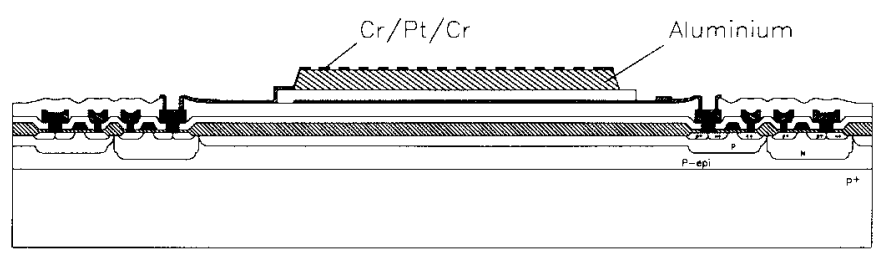

(b)

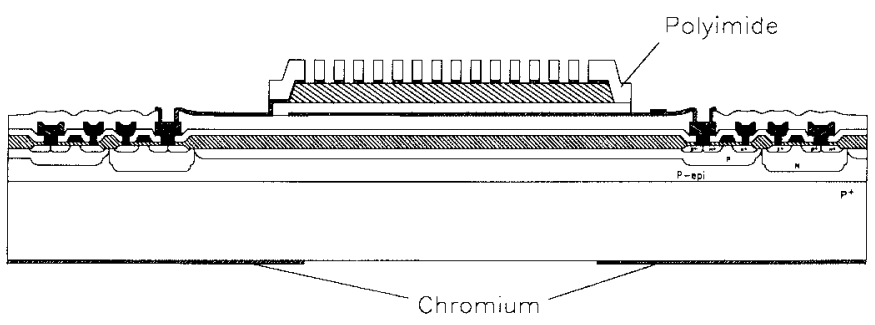

(c)

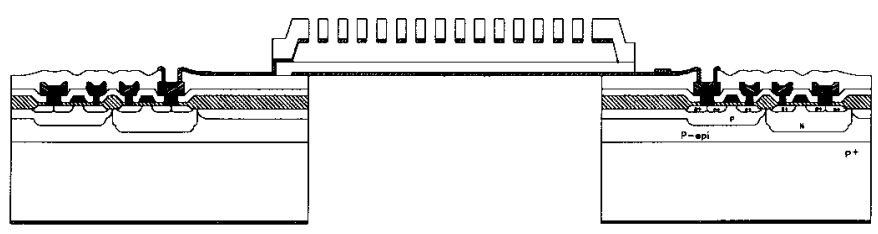

(d)

Fig. 4. Fabrication process of the integrated condenser microphone. (a) Standard CMOS processing and deposition of $\mathrm{Cr} / \mathrm{Pt} / \mathrm{Cr}$ diaphragm electrode and polyimide diaphragm. (b) Deposition of $\mathrm{Al}$ sacrificial layer and $\mathrm{Cr} / \mathrm{Pt} / \mathrm{Cr}$ backplate electrode. (c) Deposition of polyimide backplate and $\mathrm{Cr}$ etchmask on the back of substrate. (d) Etching of sacrificial layer and substrate.

a standard twin-well CMOS process [15]. The following step in the integrated sensor process [Fig. 4(a)] is to deposit and pattern the chromium/platinum/chromium diaphragm electrode on the front. This is done with liftoff using photoresist since then no metals are deposited directly onto the surface of the CMOS circuits. Second, the polyimide diaphragm layer is spun on, patterned, and cured at $300^{\circ} \mathrm{C}$. The polyimide processing is based on the use of photosensitive polyimide (HTR3-200 from OCG Microelectronics) and is similar to the procedures described previously [11]. Subsequently, the aluminum sacrificial layer is deposited and patterned [Fig. 4(b)], and since the CMOS circuits have open areas (bond pads) of aluminum, action must be taken to protect these areas during the patterning of the sacrificial layer. This is done by applying a layer of photoresist over the CMOS circuits before the deposition of the aluminum sacrificial layer. Therefore, again no metal is deposited directly on the surface of the CMOS circuits, and the photoresist layer will protect the CMOS when patterning the aluminum. After removing the protective photoresist over the CMOS circuits, the chromium/platinum/chromium backplate electrode is deposited and patterned with liftoff. This layer

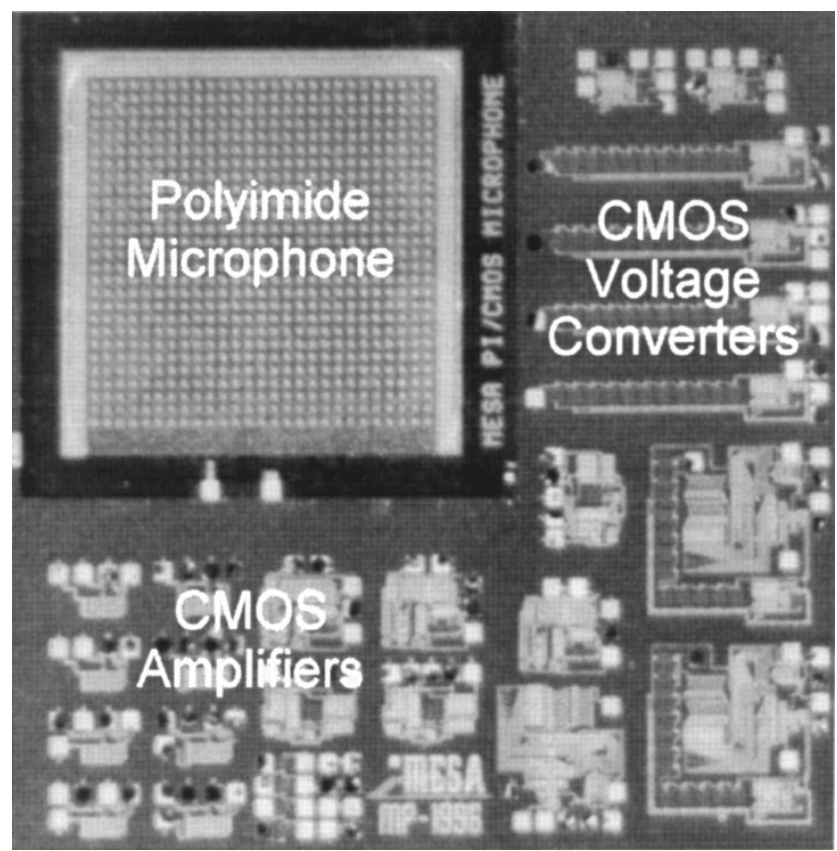

Fig. 5. Chip photograph of polyimide condenser microphone with integrated CMOS detection circuit.

may also be used to provide an electrical connection between the microphone and CMOS circuit. The polyimide backplate is then spun on, patterned, and cured, and a chromium mask layer is deposited and patterned on the back side of the substrate with liftoff [Fig. 4(c)]. The aluminum sacrificial layer is then etched in a wet chemical solution during which the CMOS is protected by a thick layer of photoresist. After the etching, the photoresist is stripped in acetone, and the structures are freezedried using a process with cyclohexane [11]. The final step to realize the structure in Fig. 4(d) is to etch the substrate under the diaphragm. This is done in an Oxford Plasma Lab 100 cryogenic reactive ion etching system with an $\mathrm{SF}_{6} / \mathrm{O}_{2}$ plasma at a temperature of $-110^{\circ} \mathrm{C}$. At this low temperature, the etch rate of silicon is considerable (up to $4 \mu \mathrm{m} / \mathrm{min}$ ) and highly anisotropic, allowing very steep sidewalls [16]. The advantage of this etch process over wet chemical etching, apart from process compatibility, is that the anisotropy of the process is not dependent on the crystal orientation, meaning that circular structures can also be realized and that alignment of the structure to the crystal directions is unnecessary.

After etching through the silicon substrate, the insulation layers from the CMOS process still remain on the diaphragm, causing it to buckle. These silicon oxide layers are removed by etching the backside of the substrate with HF vapor. In practice, this etch process is performed by placing the substrate over a container with concentrated HF (50\%). The substrate at the same time seals off the container and thereby protects the front. With this process, high-etch rates of silicon oxide of up to $250 \mathrm{~nm} / \mathrm{min}$ can be achieved. The etching stops on the diaphragm electrode because the chromium surface is inert in HF. A photograph of a completed integrated microphone is shown in Fig. 5. The electrical connections from the microphone to the electronics on this test chip are made with bond wires to facilitate measurements with different 
TABLE II

Measured Parameters of CMOS Process Control Module Before and After the Microphone Process

\begin{tabular}{lllllr}
\hline & \multicolumn{3}{c}{ NMOS } & \multicolumn{2}{c}{ PMOS } \\
& Before & After & Before & After & Unit \\
\hline Threshold voltage $\left(\mathrm{V}_{\mathrm{t}}\right)$ & 0.53 & 0.51 & 0.77 & 0.86 & $\mathrm{~V}$ \\
Transconductance $(\beta)$ & 75 & 69 & 34 & 31 & $\mu \mathrm{A} / \mathrm{V}^{2}$ \\
Bulk factor $(\gamma)$ & 0.61 & 0.61 & 0.54 & 0.53 & $\mathrm{~V}^{1 / 2}$ \\
Substrate doping density $\left(\mathrm{N}_{\text {sub }}\right)$ & 2.05 & 2.08 & 1.61 & 1.66 & $10^{16} \mathrm{~cm}^{3}$ \\
Subthreshold slope & 94 & 77 & 86 & 139 & $\mathrm{mV} / \mathrm{dec}$ \\
Junction breakdown voltage $\left(\mathrm{V}_{\mathrm{bd}}\right)$ & 16.6 & 16.9 & 17.0 & 17.0 & $\mathrm{~V}$ \\
\hline
\end{tabular}

parts of the CMOS circuitry. In the future, this connection will be made with the $\mathrm{Cr} / \mathrm{Pt} / \mathrm{Cr}$ layer used for the backplate electrode. The overall size of the chip is $5.1 \times 5.1 \mathrm{~mm}$ of which the microphone occupies approximately $3 \times 3 \mathrm{~mm}$. The microphone in Fig. 5 was not diced, however, a method for dicing was later developed using standard dicing tape on both sides of the wafer and a wafer saw.

\section{TeSt AND MEASuREMENTS}

An indication of the compatibility of the microphone fabrication process has been acquired from measurements on the process control modules (PCM's) of the CMOS process. In Table II, the measured parameters of one such module before and after the fabrication process are shown. The first conclusion to draw from these measurements is that the microphone process does not dramatically change the parameters of the CMOS components. Nevertheless, considering first the threshold voltages, a decrease for NMOS components and an increase for PMOS components were measured after the fabrication process. This suggests the presence of a trapped charge within the gate oxide. Considering the fabrication process of the microphone, some change of the parameters might be expected since the sacrificial layer in the microphone due to process equipment limitations was deposited using $e$-beam evaporation. It is known that this process produces $\mathrm{X}$-rays, which can cause trapped charges in the gate oxide [17]. This result supports the observation that e-beam evaporation, in general, should be avoided in the future, the best alternative being dc magnetron sputtering. Considering the other MOS parameters from Table II, little or no change is observed which indicates that the microphone process is fully compatible. Only the subthreshold slopes show a significant change, which is, however, directly caused by the change of the threshold voltages.

The integrated microphone was tested using the acoustical test station [18] of which the basic principle is shown in Fig. 6. First, the sensitivity of the microphone with the integrated PMOS preamplifier was measured as a function of an externally applied bias voltage. The measurements were performed at a frequency of $1 \mathrm{kHz}$ and a sound pressure level (SPL) of $100 \mathrm{~dB}$ re. $20 \mu \mathrm{Pa}$, and the results are shown in Fig. 7. The shape of the curve resembles those reported previously [11], and the critical bias voltage of the microphone was measured to be $16 \mathrm{~V}$. If the built-in dc-dc voltage converter is employed, the required external voltage is drastically reduced. In Fig. 8, the measured sensitivity versus supply voltage is shown for

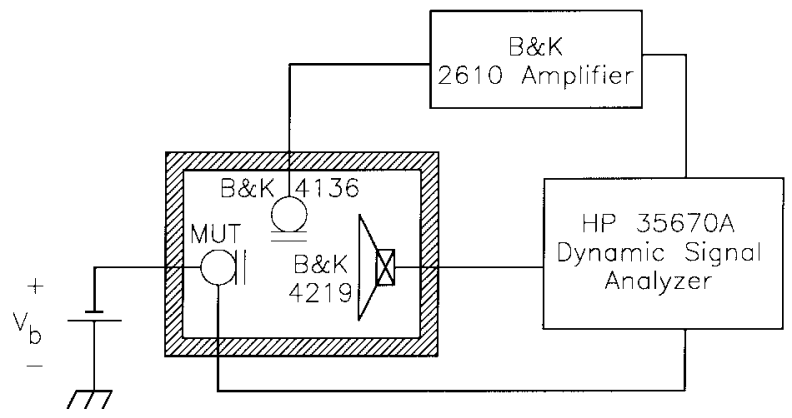

Fig. 6. Measurement setup for the acoustical characterization of the integrated microphone.

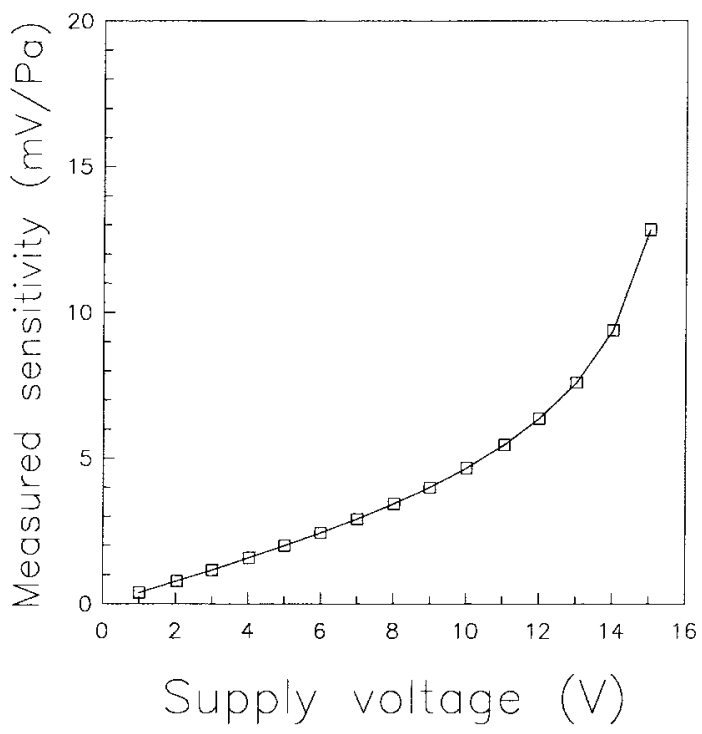

Fig. 7. Measured microphone sensitivity versus supply voltage using external biasing.

the integrated microphone using the ten-stage PMOS voltage converter. It can be seen that this curve is identical to the top half of the curve with external biasing, however, the required supply (bias) voltage is now in a range of 1.55-2 $\mathrm{V}$ instead of $8-15 \mathrm{~V}$. For supply voltages below $1.55 \mathrm{~V}$, the integrated microphone does not work since the dc-dc voltage converter does not function properly. This is due to the oscillator circuit (Fig. 2), which ceases to function as the supply voltage approaches the sum of the threshold voltages in the inverters $(\approx 1.4 \mathrm{~V})$, forcing the transistors into the subthreshold region. 


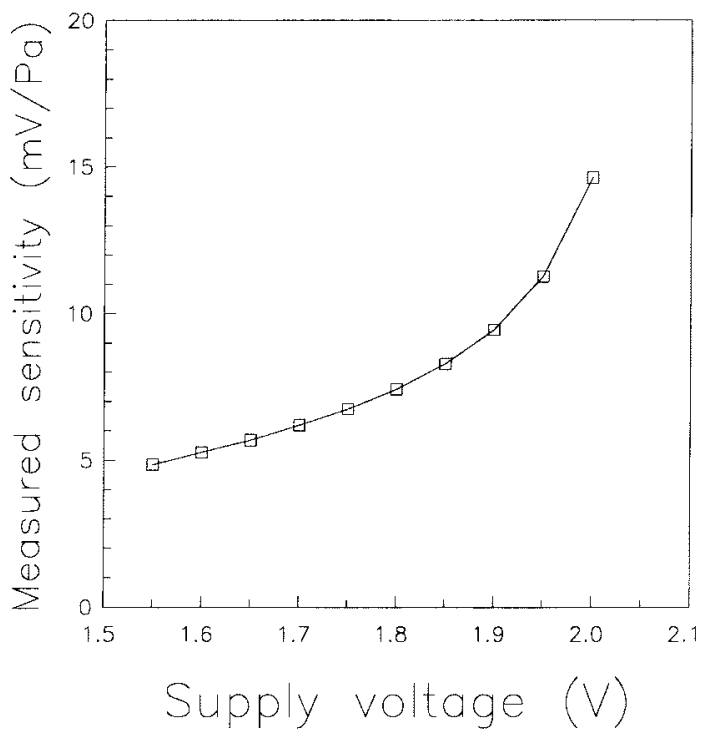

Fig. 8. Measured microphone sensitivity versus supply voltage using the internal $\mathrm{dc}-\mathrm{dc}$ voltage converter.

The frequency response of the integrated microphone with the $\mathrm{dc}-\mathrm{dc}$ voltage converter was measured at a supply voltage of $1.9 \mathrm{~V}$, corresponding to a bias voltage of $14 \mathrm{~V}$, and the results are shown in Fig. 9. The response is $-6 \mathrm{~dB}$ at $15 \mathrm{kHz}$, and the $3-\mathrm{dB}$ rolloff frequency is $8 \mathrm{kHz}$. The sensitivity at 1 $\mathrm{kHz}$ was measured to be $10.0 \mathrm{mV} / \mathrm{Pa}$. Comparing these results with the simulations (sensitivity: $21.7 \mathrm{mV} / \mathrm{Pa}$ and $f_{-3 \mathrm{~dB}}: 27$ $\mathrm{kHz}$ ), it is apparent that a difference exists between theory and measurements. Also, the measured collapse voltage (16 V), according to Fig. 7, is lower than expected from the quasi-static model $(24 \mathrm{~V})$. Together with the relatively lowrolloff frequency, this indicates that the air gap might be smaller than assumed in the simulation. Assuming an air gap of $2.9 \mu \mathrm{m}$, both the quasi-static and dynamic calculations match the measurements, regarding the collapse voltage $(17 \mathrm{~V})$ and rolloff frequency $(7.9 \mathrm{kHz})$. However, according to these simulations, the sensitivity at $1 \mathrm{kHz}$ should then be much higher $(29.1 \mathrm{mV} / \mathrm{Pa})$. This discrepancy is believed to be caused by a parasitic capacitance within the microphone structure itself. During the etching of the aluminum sacrificial layer in the microphone, a ring of material was left near the edges of the diaphragm and backplate. Since the aluminum is in direct contact with the backplate electrode and since the ring partly covers the diaphragm electrode, a large parasitic capacitance can be expected. A measurement with a Hewlett-Packard 4194A impedance analyzer reveals that the microphone capacitance is $\sim 30 \mathrm{pF}$, compared to the theoretical value of 9.4 $\mathrm{pF}$ which can be calculated from (10). Assuming a parasitic capacitance of $20 \mathrm{pF}$, which is reasonable in this situation, the simulation yields a sensitivity of $10.3 \mathrm{mV} / \mathrm{Pa}$. In the future, this parasitic capacitance can be reduced considerably by changing the dimensions of the layers in the microphone structure and/or by etching the sacrificial layer further. This should lead to sensitivities as high as $25 \mathrm{mV} / \mathrm{Pa}$ for a supply voltage of 1.9 V. A possible explanation for the reduced air gap could be that the tensile stress in the backplate electrode makes the backplate

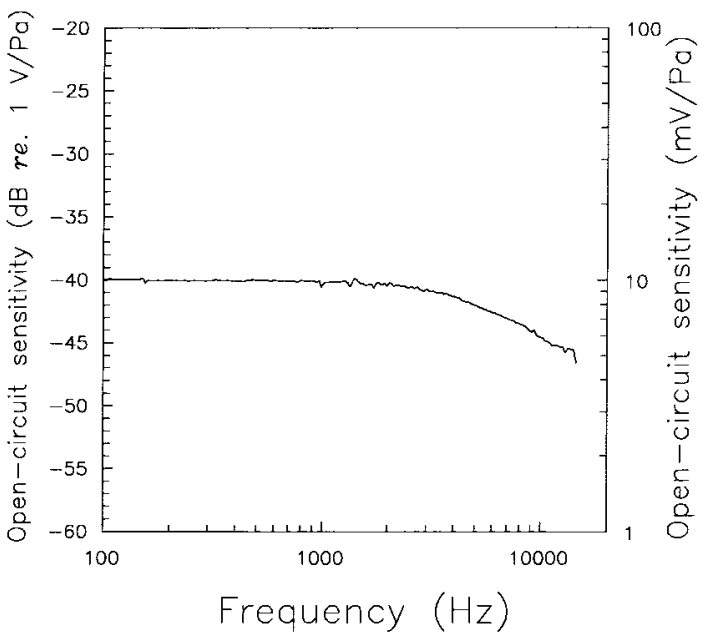

Fig. 9. Measured frequency response of the integrated microphone with a supply voltage of $1.9 \mathrm{~V}$.

deflect, which might be verified by an optical measurement of the backplate profile.

The distortion of the microphone is shown in Fig. 10 as a function of the supply voltage at a sound pressure level of $120 \mathrm{~dB}$ re. $20 \mu \mathrm{Pa}$. As previously reported [19], the distortion increases sharply as the microphone approaches the collapsed situation. For a supply voltage of $1.9 \mathrm{~V}$, the distortion is $10.1 \%$. Since $10 \%$ distortion is a typical limit of acceptable distortion, this means that if the microphone is operated at $1.9 \mathrm{~V}$, the maximum measurable sound pressure level is approximately $120 \mathrm{~dB}$ re. $20 \mu \mathrm{Pa}$. Finally, the noise of the integrated microphone was measured with no bias voltage applied to the microphone. This was done since the acoustical noise level of the surroundings of the setup was found to be dominating [ 40 dB(A) SPL]. A quieter setup will be required for acoustical noise measurements. The measured noise can therefore be considered the best possible noise level since the acoustical noise must be added. The A-weighted noise voltage in a bandwidth of $200 \mathrm{kHz}$ was found to be $4.5 \mu \mathrm{V}$. For a sensitivity of $10.0 \mathrm{mV} / \mathrm{Pa}$, this yields an equivalent noise level (ENL) of $27 \mathrm{~dB}(\mathrm{~A})$ SPL. Subtracting this value from the maximum measurable sound pressure level gives a dynamic range of $93 \mathrm{~dB}$ of the integrated microphone. The measured specifications of the integrated microphone are summarized in Table III.

\section{CONCLUSIONS AND DISCUSSION}

In this paper, an integrated condenser microphone has been described based on polyimide technology and realized by postprocessing on a CMOS wafer at low-processing temperatures. A new cryogenic reactive ion etch process for the etching of the silicon substrate has been implemented, thereby replacing any wet chemical etching of silicon. The result of this is a process which can be performed without harm to the CMOS circuits already present on the silicon substrate. Measurements on the CMOS process control modules show that the microphone process does have a minor influence on the threshold voltages of the MOS transistors. This is believed to be caused by trapped gate oxide charges generated by X-rays 
TABLE III

Measured Specifications of the Integrated Polyimide Condenser Microphone

\begin{tabular}{lll}
\hline & & Conditions \\
\hline Supply voltage & $1.9 \mathrm{~V}$ & \\
Total power dissipation & $150 \mu \mathrm{W}$ & \\
Sensitivity @ $1 \mathrm{kHz}$ & $10.0 \mathrm{mV} / \mathrm{Pa}$ & $\mathrm{SPL}=100 \mathrm{~dB}$ re. $20 \mu \mathrm{Pa}$ \\
Frequency response $( \pm 3 \mathrm{~dB})$ & $100 \mathrm{~Hz}$ to $15 \mathrm{kHz}$ & $\mathrm{SPL}=100 \mathrm{~dB}$ re. $20 \mu \mathrm{Pa}$ \\
A-weighted noise voltage & $4.5 \mu \mathrm{V}$ & $\Delta \mathrm{f}=200 \mathrm{kHz}$, No DC bias voltage \\
Equivalent noise level & $27 \mathrm{~dB}(\mathrm{~A})$ re. $20 \mu \mathrm{Pa}$ & $\Delta \mathrm{f}=200 \mathrm{kHz}$, No DC bias voltage \\
Maximum sound pressure level & $120 \mathrm{~dB}$ re. $20 \mu \mathrm{Pa}$ & THD $<10 \%$ \\
Dynamic range & $93 \mathrm{~dB}$ & \\
\hline
\end{tabular}

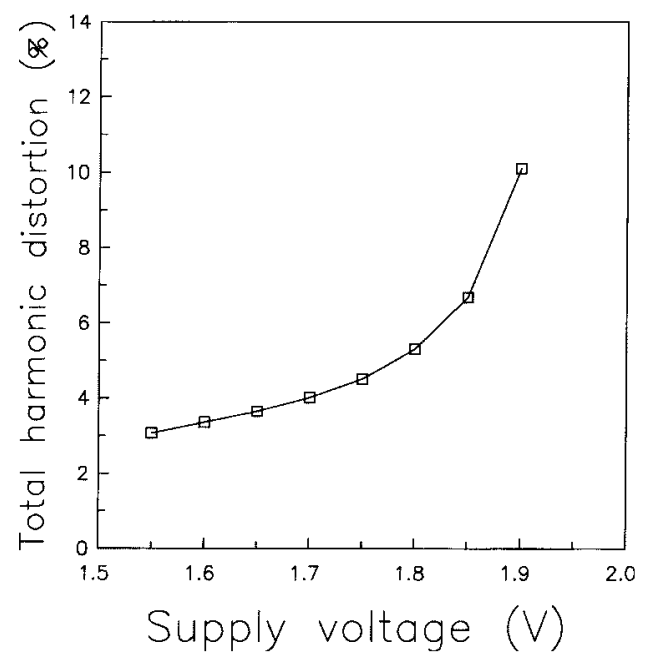

Fig. 10. Measured total harmonic distortion of the integrated microphone versus power supply voltage at a sound pressure level of $120 \mathrm{~dB}$ re. $20 \mu \mathrm{Pa}$.

from the $e$-beam evaporation of the aluminum sacrificial layer in the microphone. This problem can be avoided in the future by using a different process, such as dc magnetron sputtering, for deposition of the sacrificial layer.

The integrated microphones produced with this process prove to have properties, which are among the best reported so far for silicon condenser microphones. The inclusion of a preamplifier on the silicon substrate with the microphone has been reported previously by other authors [9], [10], and the performance of the integrated polyimide microphone is not exceptional compared to those. What makes this device exceptional is the integration of the dc-dc voltage converter, which makes the condenser microphone extremely sensitive at remarkably low-supply (bias) voltages. The devices which have been produced so far have ENL's of 26-28 dB(A) SPL for supply voltages of $1.9-2.0 \mathrm{~V}$ with no dc bias voltage applied to the microphone.

It should be noted that the devices described in this paper are among the first to be produced and that several improvements can be carried out, which can further increase the performance. As mentioned previously, the etching of the aluminum sacrificial layer should be optimized to decrease the accidental parasitic capacitance in the microphone. This single modification should lead to an increase of the sensitivity by a factor of two, which in theory should reduce the ENL to around $20 \mathrm{~dB}(\mathrm{~A})$ SPL. Further reductions of the noise and supply voltage can be achieved by applying a process for the CMOS circuits, which is optimized for low-voltage low-noise circuits. This indicates that there is still room for improvements, and judging from the results obtained so far, an integrated microphone with a noise level below $20 \mathrm{~dB}(\mathrm{~A})$ SPL and a supply voltage of $1.2 \mathrm{~V}$ is not unrealistic.

\section{ACKNOWLEDGMENT}

The authors are grateful to J. Bomer for the preparation of the samples and the IC-process group at the MESA Research Institute for providing the CMOS substrates.

\section{REFERENCES}

[1] D. Hohm and R. Gerhard-Multhaupt, "Silicon-dioxide electret transducer," J. Acoust. Soc. Amer., vol. 75, pp. 1297-1298, 1984.

[2] D. Hohm and G. Hess, "A subminiature condenser microphone with silicon nitride membrane and silicon back plate," J. Acoust. Soc. Amer., vol. 85, pp. 476-480, 1989.

[3] J. Bergqvist, F. Rudolf, J. Maisano, F. Parodi, and M. Rossi, "A silicon condenser microphone with a highly perforated backplate," in Proc. 6th Int. Conf. Solid-State Sensors and Actuators, San Francisco, CA, June, 1991, pp. 266-269

[4] W. Kühnel and G. Hess, "A silicon condenser microphone with structured back plate and silicon nitride membrane," Sens. Actuators A, vol. 30, pp. 251-258, 1992.

[5] J. Bergqvist and F. Rudolf, "A silicon condenser microphone using bond- and etch-back technology," Sens. Actuators A, vol. 45, pp. 115-124, 1994

[6] P. R. Scheeper, W. Olthuis, and P. Bergveld, "Improvement of the performance of microphones with a silicon nitride diaphragm and backplate," Sens. Actuators A, vol. 40, pp. 179-186, 1994.

[7] J. Bergqvist and J. Gobet, "Capacitive microphone with a surface micromachined backplate using electroplating technology," IEEE J. Microelectromech. Syst., vol. 3, no. 2, pp. 69-75, 1994.

[8] Q. Zou, Z. Li, and L. Liu, "Design and fabrication of silicon condenser microphone using corrugated diaphragm technique," IEEE J. Microelectromech. Syst., vol. 5, pp. 197-204, 1996.

[9] A. Kovács and A. Stoffel, "Integrated condenser microphone with polysilicon electrodes," in Proc. 6th Workshop on Micromachining, Micromechanics and Microsystems (MME'95), Copenhagen, Denmark, Sept. 1995, pp. 132-135.

[10] J. J. Bernstein and J. T. Borenstein, "A micromachined silicon condenser microphone with on-chip amplifier," in Proc. Solid-State Sensor and Actuator Workshop, Hilton Head, SC, June 1996, pp. 239-243.

[11] M. Pedersen, W. Olthuis, and P. Bergveld, "A silicon condenser microphone with polyimide diaphragm and backplate," Sens. Actuators A, vol. 63/2, pp. 97-104, 1997.

[12] J. F. Dickson, "On-chip high-voltage generation in MNOS integrated circuits using an improved voltage multiplier technique," IEEE J. SolidState Circuits, vol. SC-11, no. 3, pp. 374-378, 1976.

[13] M. Pedersen, "A polymer condenser microphone realized on silicon containing preprocessed integrated circuits," Ph.D. dissertation, Univ. Twente, The Netherlands, 1997. 
[14] _ _ "On the mechanical behavior of thin perforated plates and their application in silicon condenser microphones," Sens. Actuators A, vol. 54, pp. 499-504, 1996.

[15] A. Stolmeijer, "A twin-well CMOS process employing high-energy ion implantation," IEEE Trans. Electron Devices, vol. ED-33, pp. 450-457, 1986.

[16] J. W. Bartha, J. Greschner, M. Puech, and P. Maquin, "Low temperature etching of $\mathrm{Si}$ in high density plasma using $\mathrm{SF} 6 / \mathrm{O}_{2}$, , Microelectron. Eng., vol. 27, pp. 453-456, 1995.

[17] S. M. Sze, Semiconductor Devices, Physics and Technology. New York: Wiley, 1985, p. 367

[18] M. Pedersen, R. Schellin, W. Olthuis, and P. Bergveld, "Electroacoustical measurements of silicon microphones on wafer scale," $J$. Acoust. Soc. Amer., vol. 101, pp. 2122-2128, 1997.

[19] M. Pedersen, W. Olthuis, and P. Bergveld, "Harmonic distortion in silicon condenser microphones," J. Acoust. Soc. Amer., vol. 102, pp. 1582-1587, 1997.

Michael Pedersen was born in Holbæk, Denmark, on November 29, 1969. $\mathrm{He}$ received the M.Sc. degree in electrical engineering from the Technical University of Denmark in 1993 and the Ph.D. degree with honors in 1997 from the University of Twente, The Netherlands.

Currently, he is with Knowles Electronics, Inc., Rolling Meadows, IL. His major research interests focus on MEMS technologies applied to active and passive acoustical components.

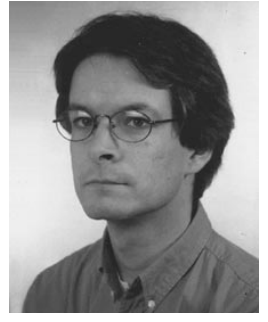

Wouter Olthuis was born in Apeldoorn, The Netherlands, on October 23, 1960. He received the M.Sc. degree in electrical engineering in 1986 and the Ph.D. degree in 1990, both from the University of Twente, The Netherlands.

Currently, he is an Assistant Professor in the Biosensor Technology Group of the MESA Institute, University of Twente.

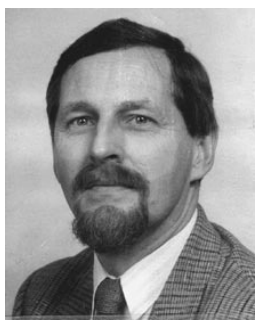

Piet Bergveld was born in Oosterwolde, The Netherlands, on January 26, 1940. He received the M.Sc. degree in electrical engineering from the University of Eindhoven, The Netherlands, in 1965 and the Ph.D. degree from the University of Twente, The Netherlands, in 1973. The subject of his dissertation was the development of ISFET' and related devices and the actual invention of the ISFET, which has also been investigated by many international research groups of universities as well as industry.

Since 1965, he has been a Member of the Biomedical Engineering Division, Department of Electrical Engineering, University of Twente, The Netherlands, where in 1984 he was appointed as Full Professor in Biosensor Technology. $\mathrm{He}$ is one of the Project Leaders at the MESA Research Institute. His research interests concern the further development of ISFET technology as well as physical sensors for biomedical and environmental applications, which has resulted in more than 200 papers. 